\title{
Benefits of Cardiovascular Rehabilitation of Insufficient Cardiac to the Institute of Cardiology of Abidjan
}

\author{
Justin Koffi Djinguin 1,2,3, N'guetta Roland3', Anderson Amani Kwadjau' \\ Ambroise Gnaba ${ }^{3}$, Jean Jacques N'Djessan ${ }^{3}$, Ines Angoran ${ }^{3}$, \\ Arnaud Ekou ${ }^{3}$, Herman $\mathrm{YaO}^{3}$, Kramoh K. Euloge ${ }^{3}$ \\ ${ }^{1}$ Institute of Cardiology of Abidjan, Abidjan, Ivory Coast \\ ${ }^{2}$ Hospital of the National Police, Abidjan, Ivory Coast \\ ${ }^{3}$ University Felix Houphouet Boigny of Cocody, Abidjan, Ivory Coast \\ Email: koffidjinguin@yahoo.fr
}

Received 1 January 2016; accepted 15 January 2016; published 19 January 2016

Copyright (C) 2016 by authors and OALib.

This work is licensed under the Creative Commons Attribution International License (CC BY). http://creativecommons.org/licenses/by/4.0/

(c) (i) Open Access

\section{Abstract}

Objective: Our work aims to propose a simplified approach by the cardiovascular rehabilitation for the cardiac heart failure. Method and Patients: We realized an observational prospective study during 12-month period which included 85 cardiac heart failure patients at Abidjan Cardiology Institute. Results: 85 patients were included in our study; the average age was $45 \pm 20$ years. The ratio-sex (men/woman) was to 2 . Six patients abandoned the study due to financial issues so that we continued the study with 79 patients. According to the stage of cardiac failure by New York Heart Association (NYHA): after the cardiac rehabilitation, the values of $P(P=0.000)$ confirmed that the rehabilitation improved considerably the potency tolerance. 60 patients $(75 \%)$ were at the NYHA II, III stages. According to potency test results, rehabilitation improved significantly the patients potency in watt of the $(P=\mathbf{0 . 0 0 0})$. According to left ventricle ejection fraction: rehabilitation improved patients cardiac output. Variability rate was $50 \%$ for the cardiac output between $20 \%-30 \%$ and $30 \%-40 \%$. Conclusion: In addition to drug therapy, cardiovascular rehabilitation is now recognized in the management of heart failure. Improvements of signs and patients' life quality remain the major benefits of Cardiovascular Rehabilitation and the reduction of hospitalizations and mortality.

\section{Keywords}

Heart Failure, Cardiovascular Rehabilitation, Abidjan, Cardiology Institute of Cardiology, Abidjan

Subject Areas: Clinical Trials, Public Health

How to cite this paper: Djinguin, J.K., Roland, N., Kwadjau, A.A., Gnaba, A., N’Djessan, J.J., Angoran, I., Ekou, A., Yao, H. and Euloge, K.K. (2016) Benefits of Cardiovascular Rehabilitation of Insufficient Cardiac to the Institute of Cardiology of Abidjan. Open Access Library Journal, 3: e2270. http://dx.doi.org/10.4236/oalib.1102270 


\section{Introduction}

L'insuffisance cardiaque (IC) est décrite comme l'une des épidémies du XXIème siècle. L'allongement de l'espérance de vie, la plus grande maîtrise des facteurs de risque cardiovasculaire, la précocité de prise en charge et le suivi plus extensif d'autres pathologies cardiaques telles que les cardiopathies ischémiques ou valvulaires, contribuent à une augmentation de la prévalence et de l'incidence de l'insuffisance cardiaque notamment au-delà de 60 ans [1].

Au cours des 25 dernières années, des progrès majeurs ont été accomplis dans la prise en charge médicamenteuse de l'IC à fraction d'éjection altérée. Le blocage du système rénine angiotensine-aldostérone (SRAA), puis du système sympathique et l'utilisation d'antagonistes des récepteurs aux minéralo-corticoïdes (ARM) ont effectivement contribué à une réduction de la morbidité et des mortalités cardiovasculaire et globale. Cependant, les derniers essais cliniques suggèrent que nous avons atteint un effet seuil et que malgré l'ajout de thérapeutiques, le gain reste minime. En effet, on assiste à une divergence en termes de mortalité et morbidité liée à cette pathologie: alors que la mortalité décroît, la fréquence des hospitalisations augmente de manière préoccupante au prix d'une altération de la qualité de vie des patients et d'un surcoût en termes de santé publique [1]. Dans ce contexte, les interventions non pharmacologiques à visée hémodynamique (resynchronisation cardiaque et défibrillateurs automatiques) et de réadaptation cardiaque ont pallié en partie au manque d'efficacité relative des stratégies médicamenteuses. Elles ont amélioré le pronostic du patient insuffisant cardiaque et font l'objet d'une validation de leurs utilisations dans les dernières recommandations de la Société Européenne de Cardiologie (ESC). De plus, l'épidémiologie même de l’IC a changé. Désormais, les patients poly-pathologiques, cumulant IC et diabète, broncho-pneumopathie chronique obstructive (BPCO), insuffisance rénale ou anémie sont sur le devant de la scène. Ces nouvelles entités obligent de plus en plus à une coordination des professionnels de santé autour de "l'acteur patient" [1]. Ainsi se développent des programmes d'éducation thérapeutique, afin de pallier aux insuffisances des thérapeutiques actuelles et de prendre en compte le patient dans sa globalité. L'idée est de permettre une amélioration de la prise en charge du patient grâce à de nouvelles approches, au-delà de la pharmacologie et des techniques interventionnelles. Même si l'éducation thérapeutique du patient (ETP) a fait l'objet de recommandations des sociétés savantes depuis plusieurs années, trop peu de patients en bénéficient à ce jour. Des arguments d'ordre matériel (manque de temps, isolement géographique), médical (manque de formation et de connaissances) et économique (financement) semblent en être les principales raisons [2]. Ainsi, les cliniciens peinent à intégrer la réadaptation cardiovasculaire, dont ils connaissent pourtant les bénéfices à leur pratique quotidienne [3]. Dans ce contexte, notre travail vise à proposer une approche simplifiée de la réadaptation cardiovasculaire pour l'Insuffisance Cardiaque afin de faciliter son utilisation et sa diffusion, auprès de médecins cardiologues exerçant l'Institut de cardiologie et sur tout le territoire de la Côte d'Ivoire et dans les autres pays Africains ou la réadaptation cardiovasculaire est très peu pratiquée.

\section{Patients et Méthode}

Nous avons réalisé une étude prospective observationnelle sur une période de 12 mois qui a inclus 85 patients admis pour Insuffisance Cardiaque à l'Institut de cardiologie d'Abidjan.

Les critères d'inclusion sont la régression des signes périphériques d'insuffisance cardiaque (absence d'œdème, régression, l'absence de thrombus visualisé à l'échographie cardiaque, régression de la dyspnée à partir du stade initial, régression de l'hépatomégalie) la dysfonction du ventricule gauche (FE $\leq 40$ ), l'absence d'hypertension artérielle pulmonaire sévère, l'absence de cardiopathie obstructive grave.

Les critères d'exclusions étaient toutes les comorbidités pouvant empêcher la régression des signes ou aggravées le tableau clinique du patient à savoir l'insuffisance rénale chronique, un trouble du rythme grave, un accident vasculaire cérébral, un thrombus intra cavitaire, une hypertension artérielle pulmonaire sévère, une impotence fonctionnelle, une cardiopathie obstructive.

L'étude a consisté à la réalisation d'une épreuve d'effort comme élément essentiel pour la prescription d’un programme de réadaptation cardiovasculaire.

Pour tous les patients, nous avons utilisé le même protocole; 25 Watts toutes les 2 minutes malgré les aptitudes physiques différentes.

L'épreuve d'effort était symptômes dépendant malgré le protocole commun.

Chaque patient a eu comme programme une épreuve d'endurance (tapis roulant ou ergomètre à raison de trente minutes par séance soit trois séances par semaine au total 144 séances pendant le suivi), une épreuve de gym- 
nastique (trente minutes par séance soit 144 séances pendant tout le suivi), une épreuve de marche encadrée de 45 minutes soit deux fois par séance au total 96 séances. Ces patients ont également bénéficié d’un diagnostic éducatif qui consistait en un entretien individuel et/ou collectif de compréhension des besoins éducatifs du patient, avec une fréquence de 2 séances par semaine soit un total de 96 séances.

Un entretien avec le psychologue par mois soit au total 12 séances. Les principaux thèmes abordés étaient: le patient insuffisant cardiaque et sa maladie, à savoir: la maladie chronique du patient: son vécu et ses représentations, le retentissement de la maladie sur la vie du patient, l'évaluation des connaissances du patient sur les signes spécifiques, les facteurs déclenchant ou aggravants, les éléments de suivi au long cours de l'insuffisance cardiaque, le traitement de l'insuffisance cardiaque, le patient insuffisant cardiaque et sa diététique, le patient insuffisant cardiaque et son activité physique.

Les paramètres étudiés au cours de cette étude sont: la pression artérielle exprimée en Mmhg, l'essoufflement estimé à partir de la NYHA, l'aspect physique du patient apprécié à partir du poids du patient exprimé en Kilogramme, la fraction d'éjection du ventricule gauche exprimée en pourcentage, la fréquence cardiaque exprimée en battement par minute.

Pour cette étude, la méthode de Karvonen a été utilisée pour déterminer la fréquence cardiaque d'entraînement du patient. Le pic de consommation d'oxygène n’a pu être déterminé.

La première évaluation a eu lieu après douze mois de prise en charge (d'activité physique associée au traitement médical de l'insuffisance cardiaque). Elle a consisté à une réévaluation de l'aptitude physique, de l'épreuve d'effort et de l'échographie cardiaque.

Methode D’Analyse Statistique: les tests de proportion ont été réalisés à l’aide du logiciel Stata 9.0.

\section{Resultats}

A l'inclusion, 85 patients ont été retenus dans notre étude.

L'âge moyen de nos patients était de 45 ans \pm 20 ans avec un sex-ratio (homme/femme) de 2. Six patients soit 7\% ont abandonné les séances de réadaptation parce que les jugeant contraignant et coûteux. Nous avons poursuivi l'étude avec 79 patients.

Selon le stade de l’Insuffisance cardiaque à travers la NYHA.

\begin{tabular}{cccccc}
\hline \multirow{2}{*}{ STADE } & PERIODE & Avant réadaptation & Après réadaptation & Effectif & P \\
\cline { 2 - 5 } & & $15(20 \%)$ & $5(25 \%)$ & 20 & 0.0455 \\
Stage I & $40(50 \%)$ to total $100 \%$ & $5(11 \%)$ & 45 & 0.0000 \\
Stage II & $20(25 \%)$ & 0 & 20 & - \\
Stage III & $4(5 \%)$ & 0 & 4 & - \\
\hline
\end{tabular}

Après la réadaptation cardiaque les valeurs de $\mathrm{P}$ confirment que la réadaptation a amélioré considérablement la tolérance à l'effort. 60 patients soit 75\% étaient au stade II et III de la NYHA ont une bonne tolérance à l'effort après la réadaptation cardiaque réalisée à l’Institut de cardiologie d'Abidjan.

Selon les résultats de l'épreuve d'effort.

\begin{tabular}{|c|c|c|c|c|c|}
\hline EFFECTIS & PERIODE & Avant réadaptation (watt) & Après réadaptation (watt) & Effectif & $\mathrm{P}$ \\
\hline & 10 & $25(25 \%)$ & 75 (75\%) & 100 & 0.0000 \\
\hline & 29 & 50 (33\%) & $100(67 \%)$ & 150 & 0.0001 \\
\hline & 40 & 75 (33\%) & $150(25 \%)$ & 225 & 0.0000 \\
\hline
\end{tabular}

La réadaptation a amélioré de façon significative la puissance en watt des patients.

Selon la fraction d'éjection. 


\begin{tabular}{ccccccc}
\hline \multirow{2}{*}{$\begin{array}{c}\text { FRACTION } \\
\text { D’EJECTION }\end{array}$} & PERIODE & \multicolumn{2}{c}{ Avant réadaptation } & \multicolumn{2}{c}{ Après réadaptation } & Taux de variation (\%) \\
\cline { 2 - 6 } & & Intervalle & Centre de classe & Intervalle & Centre de classe & \\
& FE 1 & {$[20-30]$} & 25 & {$[35-40]$} & 37,5 & 50 \\
FE 2 & {$[30-40]$} & 35 & {$[50-55]$} & 52,5 & 50 \\
FE 3 & {$[40-50]$} & 45 & {$[60-70]$} & 65 & 44 \\
\hline
\end{tabular}

La réadaptation a amélioré la fraction d'éjection de nos patients de nos patients.

\section{Discussion}

\subsection{Bénéfices Physiologiques}

Les mécanismes expliquant l'intolérance à l'effort dans l'insuffisance cardiaque ne sont pas uniquement liés à la dysfonction ventriculaire gauche mais aussi à des anomalies complexes ventilatoires, musculaires squelettiques, endothéliales et neuro hormonales. Cela explique que la FEVG est mal corrélée à la capacité physique des sujets atteints d'insuffisance cardiaque.

L'entrainement physique, de façon similaire, n'agit pas seulement au niveau hémodynamique central, mais produit également des adaptations périphériques multiples [3].

\subsection{Capacité Physique}

La capacité physique mesurée à partir de la puissance en watts lors du test d'effort atteint environ $40 \%$ dans notre étude alors que des études plus récentes ayant utilisé la mesure du pic de $\mathrm{V}_{2}$ lors d'un effort montre que l'augmentation du pic de $V \dot{o}_{2}$ après un effort physique aérobie standard atteint environ $15 \%(2.1 \mathrm{ml} / \mathrm{kg} / \mathrm{min})$ [4].

Dans les deux cas nous notons une augmentation significative des paramètres après réadaptation cardiaque.

\subsection{Adaptations Hémodynamique Centrales}

Les données concernant l'amélioration de la FEVG, à l'effort sont démontrées dans les données d'une mataanalyse [3] [4].

Dans notre étude la réadaptation a permis d'améliorer la FEVG. Ce qui est en conformité avec les études suscitées.

\subsection{Bénéfices Cliniques}

Pendant toute la période d'étude tous les patients n’ont présenté aucuns signes nécessitant une hospitalisation. Tous nos patients ont bien adhéré au programme proposé au cours de l'étude. Cela s'expliquait par le fait qu'ils étaient fatigués des hospitalisations fréquentes à l'Institut de Cardiologie, pour eux c'étaient la solution d'espoir. A la fin de l'étude, tous les patients avaient une qualité de vie améliorée, certains ont pu reprendre leurs activités.

La réadaptation cardiaque n’a eu aucun effet délétère, plutôt elle a permis la réinsertion des patients dans la société.

Les soixante-dix-neuf patients ont réalisé tous les entrainements.

Jusqu'en 2009, les données concernant les bénéfices cliniques potentiels de l'entrainement physique provenaient de méta-analyse dont les résultats semblaient montrer une tendance à la diminution de la mortalité et des hospitalisations [5] [6].

Une très grande étude randomisée contrôlée d'entrainement physique chez les patients insuffisants cardiaques "HF-ACTION", a été organisée dans 135 centres de trois pays (Etats Unis, Canada et France). Un total de 2331 patients insuffisants cardiaques stables d'un age moyen de 60 ans a été inclus, majoritairement en classe fonctionnelle NYHA II et III avec une fraction d'éjection ventriculaire gauche moyenne de $25 \%$. L'entrainement physique était initialement supervisé dans un centre de réadaptation puis les patients étaient autonomes à domicile et devaient effectuer 5 séances de 40 minutes par semaines de vélo stationnaire ou de marche à une intensité modérée (60\% à $70 \%)$ de la réserve de fréquence cardiaque). Les patients d'entrainement physique ont montré 
une augmentation significative de leur pic de $\mathrm{Vo}_{2}$ de $4 \%$ en comparaison avec le groupe contrôle. Au terme du suivi moyen de 2.5 ans une discrète réduction de $10 \%$ du critère de jugement combinant mortalité et hospitalisation de toute cause a été observée [7] [8]. Par ailleurs, l'état de santé générale et la qualité de vie des patients ont été aussi améliorés [9] [10]. L’entrainement physique n’a pas montré d'effets délétères.

\section{Conclusion}

En complément des traitements médicamenteux, la réadaptation cardiaque a eu une place reconnue dans la prise en charge de l'insuffisance cardiaque chronique à l'Institut de Cardiologie. Elle améliore les symptômes et la qualité de vie des patients. Ceci, grâce à son action bénéfique significative sur la tolérance à l'effort, sur la puissance en Watt et la fraction d'éjection des patients.

\section{References}

[1] Hunt, S.A., Abraham, W.T., Chin, M.H., Feldman, A.M., Francis, G.S., Ganiats, T.G., Jessup, M., Konstam, M.A., Mancini, D.M., Michl, K., Oates, J.A., Rahko, P.S., Silver, M.A., Stevenson, L.W. and Yancy, C.W. (2009) Focused Update Incorporated into the ACC/AHA 2005 Guidelines for the Diagnosis and Management of Heart Failure in Adults: A Report of the American College of Cardiology Foundation/American Heart Association Task Force on Practice Guidelines: Developed in Collaboration with the International Society for Heart and Lung Transplantation. Circulation, 119, 391-479. http://dx.doi.org/10.1161/CIRCULATIONAHA.109.192065

[2] McMurray, J.J., Adamopoulos, S., et al. (2012) ESC Guidelines for the Diagnosis and Treatment of Acute and Chronic Heart Failure 2012: The Task Force for the Diagnosis and Treatment of Acute and Chronic Heart Failure 2012 of the European Society of Cardiology. Developed in Collaboration with the Heart Failure Association (HFA) of the ESC. European Journal of Heart Failure, 14, 803-869. http://dx.doi.org/10.1093/eurjhf/hfs105

[3] Downing, J. and Balady, G.J. (2011) The Role of Exercise Training in Heart Failure. Journal of the American College of Cardiology, 58, 561-569. http://dx.doi.org/10.1016/j.jacc.2011.04.020

[4] Keteyian, S.J. (2011) Exercise Training in Congestive Heart Failure: Risks and Benefits. Progress in Cardiovascular Diseases, 53, 419-428. http://dx.doi.org/10.1016/j.pcad.2011.02.005

[5] Smart, N. and Marwick, T.H. (2004) Exercise Training for Patients with Heart Failure: A Systematic Review of Factors That Improve Mortality and Morbidity. American Journal of Medicine, 116, 693-706. http://dx.doi.org/10.1016/j.amjmed.2003.11.033

[6] O’Connor, C.M., Whellan, D.J., Lee, K.L., Keteyian, S.J., Cooper, L.S., Ellis, S.J., Leifer, E.S., Kraus, W.E., Kitzman, D.W., Blumenthal, J.A., Rendall, D.S., Miller, N.H., Fleg, J.L., Schulman, K.A., McKelvie, R.S., Zannad, F. and Pina, I.L. (2009) Efficacy and Safety of Exercise Training in Patients with Chronic Heart Failure: HF-Action Randomized Controlled Trial. JAMA, 301, 1439-1450. http://dx.doi.org/10.1001/jama.2009.454

[7] Flynn, K.E., Pina, I.L., Whellan, D.J., Lin, L., Blumenthal, J.A., Ellis, S.J., Fine, L.J., Howlett, J.G., Keteyian, S.J., Kitzman, D.W., Kraus, W.E., Miller, N.H., Schulman, K.A., Spertus, J.A., O’Connor, C.M. and Weinfurt, K.P. (2009) Effects of Exercise Training on Health Status in Patients with Chronic Heart Failure: HF-Action Randomized Controlled Trial. JAMA, 301, 1451-1459. http://dx.doi.org/10.1001/jama.2009.457

[8] Piepoli, M.F., Conraads, V., Corra, U., Dickstein, K., Francis, D.P., Jaarsma, T., McMurray, J., Pieske, B., Piotrowicz, E., Schmid, J.P., Anker, S.D., Solal, A.C., Filippatos, G.S., Hoes, A.W., Gielen, S., Giannuzzi, P. and Ponikowski, P.P. (2011) Exercise Training in Heart Failure: From Theory to Practice. A Consensus Document of the Heart Failure Association and the European Association for Cardiovascular Prevention and Rehabilitation. European Journal of Heart Failure, 13, 347-357. http://dx.doi.org/10.1093/eurjhf/hfr017

[9] Wisloff, U., Stoylen, A., Loennechen, J.P., Bruvold, M., Rognmo, O., Haram, P.M., Tjonna, A.E., Helgerud, J., Slordahl, S.A., Lee, S.J., Videm, V., Bye, A., Smith, G.L., Najjar, S.M., Ellingsen, O. and Skjaerpe, T. (2007) Superior Cardiovascular Effect of Aerobic Interval Training versus Moderate Continuous Training in Heart Failure Patients: A Randomized Study. Circulation, 115, 3086-3094. http://dx.doi.org/10.1161/CIRCULATIONAHA.106.675041

[10] Ribeiro, J.P., Chiappa, G.R., Neder, J.A. and Frankenstein, L. (2009) Respiratory Muscle Function and Exercise Intolerance in Heart Failure. Current Heart Failure Reports, 6, 95-101. http://dx.doi.org/10.1007/s11897-009-0015-7 